\title{
Pathobiology of cervical spondylotic myelopathy
}

\author{
Spyridon K. Karadimas • Georgios Gatzounis • \\ Michael G. Fehlings
}

Received: 12 November 2013/Revised: 20 February 2014 / Accepted: 20 February 2014/Published online: 14 March 2014

(C) The Author(s) 2014. This article is published with open access at Springerlink.com

\begin{abstract}
In this narrative review, we aim to outline what is currently known about the pathophysiology of cervical spondylotic myelopathy (CSM), the most common cause of spinal cord dysfunction. In particular, we note the unique factors that distinguish it from acute spinal cord injury. Despite its common occurrence, the reasons why some patients develop severe symptomatology while others have few or no symptoms despite radiographic evidence confirming similar degrees of compression is poorly understood. Neither is there a clear understanding of why certain patients have a stable clinical myelopathy and others present with only mild myelopathy. Moreover, the precise molecular mechanisms which contribute to the
\end{abstract}

S. K. Karadimas · M. G. Fehlings

Institute of Medical Sciences, University of Toronto, Toronto, ON, Canada

S. K. Karadimas · M. G. Fehlings

Spinal Program, Division of Genetics and Development,

Krembil Neuroscience Centre, Toronto Western Research

Institute, University Health Network, Toronto, ON M5T 2S8,

Canada

G. Gatzounis

Department of Neurosurgery, University of Patras, 26500 Patras, Greece

M. G. Fehlings

Division of Neurosurgery, Department of Surgery, University of Toronto, Toronto, ON M5T 2S8, Canada

M. G. Fehlings $(\bowtie)$

Gerald and Tootsie Halbert Chair in Neural Repair and Regeneration, Toronto Western Hospital, University Health Network, West Wing, 4th Floor, Room 4W-449, 399 Bathurst Street, Toronto, ON M5T 2S8, Canada

e-mail: Michael.Fehlings@uhn.on.ca

URL: http://www.drfehlings.ca/ pathogenesis of the disease are incompletely understood. The current treatment method is decompression of the spinal cord but a lack of clinically relevant models of CSM have hindered the understanding of the full pathophysiology which would aid the development of new therapeutic avenues of investigation. Further elucidation of the role of ischemia, currently a source of debate, as well as the complex cascade of biomolecular events as a result of the unique pathophysiology in this disease will pave the way for further neuroprotective strategies to be developed to attenuate the physiological consequences of surgical decompression and augment its benefits.

Keywords Spinal cord - Cervical spondylotic myelopathy $\cdot$ CSM $\cdot$ Spinal cord injury

\section{Introduction}

Cervical spondylotic myelopathy (CSM), wherein the cervical cord is compressed by degenerative changes in the spine, is the commonest cause of spinal cord impairment worldwide, although precise incidence and prevalence data are lacking [1-3]. Since Stookey's original description of this condition in 1928 [4], much has been learned regarding the natural history, imaging features, treatment options and pathobiology. However, much remains to be learned regarding the molecular mechanisms of neural degeneration in this condition.

Until recently, most of the review articles related to the pathogenesis of CSM have dealt with the pathophysiological and genetic factors [5] which contribute to the development of the static and dynamic mechanical compression. It has been widely accepted that the static mechanical forces result in direct injury to the neuronal population and 
glial cells. In addition, the dynamic repetitive injuries delivered to the spinal cord during flexion and extension of the cervical spine further stretch the axons and make them more vulnerable to secondary injury. However, this simple mechanistic explanation has failed to explain the intriguing clinical presentation of CSM which includes the loss of manual dexterity, disrupted locomotion and sensory changes. Moreover, the reasons why some patients develop severe symptomatology while others have few or no symptoms despite radiographic evidence confirming similar degrees of compression are poorly understood. Neither is there a clear understanding of why certain patients have a stable clinical myelopathy and others present with only mild myelopathy. Moreover, the precise molecular mechanisms which contribute to the pathogenesis of the disease are incompletely understood.

The present review has three main goals: (1) to emphasize that CSM is a unique disease with distinguishing pathophysiological and pathobiological mechanisms from traumatic spinal cord injury (SCI), (2) to present the emerging knowledge regarding the molecular and cellular mechanisms of CSM and finally (3) to acknowledge and highlight the fact that critical questions regarding this common disease remain largely unexplored.

\section{Unique pathophysiological features}

\section{Static and dynamic mechanical factors}

Others have provided good description and analysis of the static and dynamic mechanical stress forces that operate in CSM [4, 5]. A detailed description of the biomechanical and anatomical factors that result in compression of the cervical spinal cord in CSM is beyond the scope of the present article. However, it is important to emphasize the following points which establish CSM as unique and distinct from SCI from a pathophysiologal perspective. Briefly, in CSM (in contrast to traumatic SCI):

1. There is no acute mechanical insult;

2. There is an absence of hemorrhagic necrosis in the cord;

3. The chronic and progressive nature of the disease likely induces compensatory mechanisms within the cord.

Ischemia

Ischemia was first reported as one of the crucial pathophysiological mechanisms in CSM by brain [6]. Evidence stemming from animal and human studies supports this theory. Specifically, pathological changes such as vessel wall thickening and hyalinization have been identified in the anterior spinal artery and parenchymal arterioles [6, 7] while the radiculomedullary arterial diameter is compromised by stenosis of the intervertebral foramina in patients with CSM [8]. Additional histopathological indications of ischemic injury in the gray and white matter of the cord have been observed in patients with CSM [9, 10]. Findings from a cadaveric study, where terminal branches of the anterior spinal artery and penetrating branches of the lateral pial plexus were observed to be curved and stretched around degenerative spinal spondylo-osteophytes, support the suggestion of compromised blood flow to axonal pathways including the corticospinal tracts [10]. Experimental work has provided indirect evidence suggesting ischemia in animals suffering from CSM in that angiography studies in animal models suffering from CSM revealed signs of compromised perfusion. Other investigators have studied the combined insult of direct compression and ischemia to the cord. Overall, ischemia seems to heighten the insult of cord compression [11], and changes in blood flow to the spinal cord [12]. In this setting, the corticospinal tract is the most affected part of cord [11], which fits the clinical presentation in CSM [5]. In one other experimental protocol, the direct compression of specific spinal arteries caused diminished blood flow to the respective arteries feeding that part of the spine [13]. Taylor suggested that radiculomedullary arteries supplying the cervical spinal cord were compromised by stenosis of the intervertebral foramina [8]. A pathophysiologic explanation is that age-related degenerative changes in the cervical spine can compress major feeding arteries like the vertebral artery [14], anterior spinal artery and its ventral branches, or the radicular arteries of the neuroforamina [7, 15]. As a result, the blood flow velocity within vertebral arteries can be pathologically reduced [16] while perfusion to key parts of the spinal cord is compromised [16]. Moreover, spondylotic deformations of the cervical spine such as kyphosis have been identified to be involved in the onset or the progression of the disease by interfering with the spinal cord vascular network $[17,18]$. This necessitates the careful evaluation not only of the cervical alignment but also of the global spinal alignment before the appropriate surgical approach will be decided [19].

In a review paper, Baptiste and Fehlings give a detailed description of their hypothesis regarding how the anterior and posterior chronic compression may result in decreased spinal cord blood flow and alter the spinal cord macro- and microvessel architectures [4]. However, convincing experimental validation of cord ischemia in the setting of CSM is surprisingly lacking [20].

In the past, the lack of a reliable animal model of induced CSM has led to uncertainty regarding the role of ischemia in CSM, but also of the existence of 
microvasculature distortion under chronic and progressive compression of the cervical spinal cord. Using a series of arguments, different groups have expressed their uncertainty as to whether ischemia is a crucial physiological event in CSM. Specifically, a number of clinical and experimental protocols demonstrate that patients or laboratory animals with moderate CSM have no [21] or only mild signs of ischemia $[22,23]$ while pathologic evidence of ischemia is found only when severe canal stenosis is coexisting [24, 25]. Moreover, some experimental studies found only minor changes in blood flow during compression and decompression [26]. In addition, another group using a computational model of cervical spinal cord compression predicted no compromise of blood flow in the intramedullary blood vessels. From a molecular standpoint, one of the principal arguments against the significant pathobiological role of ischemia in CSM is that ischemia, despite eliciting an apoptotic response, usually leads to cellular death via necrosis and not through the apoptotic pathway. Evidence indicating that the oligodendrocyte and neuronal cell death, that has been demonstrated in human and experimental studies, appears to be a result of apoptosis has fuelled the argument against the role of ischemic injury in CSM from some groups.

Summarizing, some of the above-described studies display significant merit, however, they present with critical limitations, which means that we do not yet have the answer regarding the role for chronic ischemia in CSM. Most of these studies have been performed in animal models where the compression is acute or sub-acute in nature and, therefore, fail to reproduce the chronic and progressive compression seen in human CSM. Moreover, many of these models entail compression not in the cervical spinal cord, but in the thoracic area. These limitations leave a gap necessitating the study of the spinal cord blood flow in experimental models which are characterized by slow progressive compression at the C5-C6 level [27, 28].

\section{Secondary to chronic compression: pathobiological events}

Endothelial cell impairment and blood spinal cord barrier disruption

Although the change in architecture of the spinal cord microvasculature was first considered 65 years ago as one of the main pathobiological phenomena in CSM, the structural and functional integrity of the spinal cord microvascular network had not been examined at the basic science level until recently. Specifically, the recent development of rat and mouse models of chronic and progressive compression of the cervical spinal cord which accurately mirror the pathophysiological characteristics and the clinical picture of the human disease has, for first time, given researchers the opportunity to clarify spinal cord microvasculature dysfunction in experimental settings. Specifically, our group has shown that the static and dynamic mechanical forces applied in a chronic and progressive fashion to the cervical spinal cord lead to disruption of the spinal cord microvasculature system which was characterized by decreased number of vessels as indicated by the decreased staining for laminin, a protein of the basement membrane of vessels (Fig. 1) [27].

Endothelial cells, the cells lining the internal surface of the blood vessels, play a critical role in the integrity of circulation from large vessels to small capillaries. Karadimas et al. showed recently that the number of endothelial cells is decreased in experimental CSM. Endothelial cells represent an important component of the blood-spinal cord barrier (BSCB) and their loss under chronic compression is critical for its integrity. Moreover, since endothelial cells have unique functions in critical aspects of vascular biology such as inflammation as well as functioning as a sensor in shear stress, their loss further promotes inflammation and vasculogenic dysfunction in the chronically compressed spinal cord parenchyma. There has been a paucity of insights regarding the molecular cascades implicated in endothelial cell function in CSM. Therefore, identifying the molecular mechanisms that are implicated in endothelial cell function under chronic compression is of crucial importance.

BSCB compromise is associated with ischemia through direct endothelial damage and inflammatory potentiation originating from hypoxic cell death in the parenchyma. Besides the endothelial cells BSCB also includes tight junction proteins, basal lamina, pericytes and astrocytic end feet. BSCB permeability in CNS disease or injury promotes edema formation into the spinal cord parenchyma and allows the entrance of inflammatory cells derived from the peripheral circulation into the spinal cord parenchyma. Recent data indicated ongoing permeability in CSM rats as well as in chronic human CSM cases [27, 29]. Importantly, the molecular mechanisms of endothelial cells survival and chronic BSCB disruption in CSM are poorly understood. After acute injury insult in the central nervous system, BSCB loose its integrity for a long period of time, however, after this period, it is restored [30, 31]. Matrix metalloproteinases (MMPs) [32, 33] and cytokines such as IL-1 $\beta$ and TNF- $\alpha$ [34] are responsible for the acute increase in vasculature permeability. Together, the loss and dysfunction of endothelial cells in acute $\mathrm{SCI} /$ stroke results in BSCB permeability, which is typically resolved several weeks after injury in animal models [30, 31, 35]. It was found that BSCB remain disrupted at chronic stages in rodent CSM models and in human CSM cases, suggesting a 


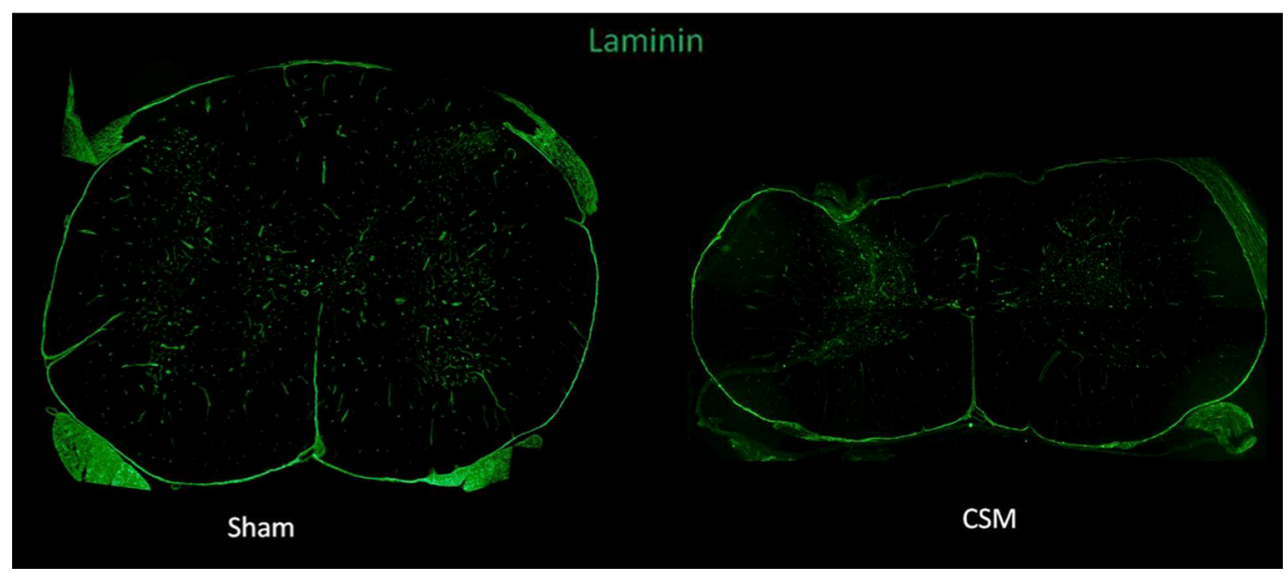

Fig. 1 Low magnification confocal images of axial spinal cord sections stained with laminin (green) demonstrate significant reduction in the number of vessels in the CSM section when compared to sham

unique series of molecular mechanisms involved in BSCB integrity in CSM.

As evidence suggests that endothelial cell dynamics are central to BSCB permeability [36], this has been a focus of ongoing experimental work examining BSCB disruption in CSM. However, other components of the neurovascular unit, such as astrocytes, should be a focus for future studies.

\section{Role of inflammation}

The inflammatory process in CSM is likely quite unique because it can be divided into two different components: (1) the inflammatory reaction which is directly derived from the mechanical compression of the spinal cord and (2) the ongoing inflammatory response induced by chronic ischemia. It could be hypothesized that the inflammatory reaction triggered by the mechanical forces take place initially. However, as recently proved, the mechanical stretch applied on the cervical spinal cord reduces the number of endothelial cells and alters the spinal cord microvasculature architecture. These two processes are happening at the same time and it is likely that both of them contribute synergistically to the progression of neurodegeneration in CSM. In the brain, post-ischemic inflammation has beneficial as well as deleterious effects. It is of high importance to shed further light on the mechanisms and factors involved here.

Until 2011, the inflammatory response elicited by the chronic-ongoing compression of the cervical spinal cord had not been characterized. Recently, our group showed that endogenous as well as systemic inflammatory cells exist early and late after the onset of the compression in human CSM cases confirming an ongoing inflammatory reaction under chronic compression [37]. Specifically, they profiled the inflammatory response during the course of the disease by performing immunohistochemistry for resident microglia/macrophages, lymphocytes and neutrophils in human CSM spinal cord tissue [37]. They demonstrated that the predominant inflammatory cell type in both early and late phases of compression is activated macrophages/ microglia [37]. However, they also observed increased numbers of myeloperoxidase-positive cells at 6 weeks after the diagnosis of the disease, indicating neutrophil infiltration in the early phases of the compression. Moreover, numerous lymphocytes were observed in the same temporal scale.

In line with the human studies, a series of experimental studies demonstrated that the inflammatory reaction generated in CSM was mostly composed of activated microglia/macrophages. However, activated macrophages/ microglia has both deleterious and protective qualities after spinal cord trauma and after acute ischemic insult. In the central nervous system (CNS), neurons express fractalkine (CX3CL1) on their membranes, while its receptor (CX3CR1) is highly expressed on microglia [38]. Based on evidence from other CNS disorders, we may expect that CX3CR1/L1 interactions are an important component of neuroinflammation; however, the role is disease specific [39-41]. Importantly, it has been demonstrated that ischemia leads to CX3CR1/L1-mediated microglia activation [42] which potentially establishes the CX3CR1/L1 pathway as a critical mechanistic link between chronic hypoxia and neuroinflammation in CSM. In a recent study, CX3CR1-deficient CSM mice were found to demonstrate decreased levels of microglia/macrophage activation which was strongly associated with decreased levels of proinflammatory cytokines/chemokines such as G-CSF, IP10, M-CSF and MIG [29]. Increased neuronal and oligodendrocyte preservation, as well as increased gray and white matter preservation, was found in the CXCR1 KO CSM mice. Finally, attenuated progression of the disease and 

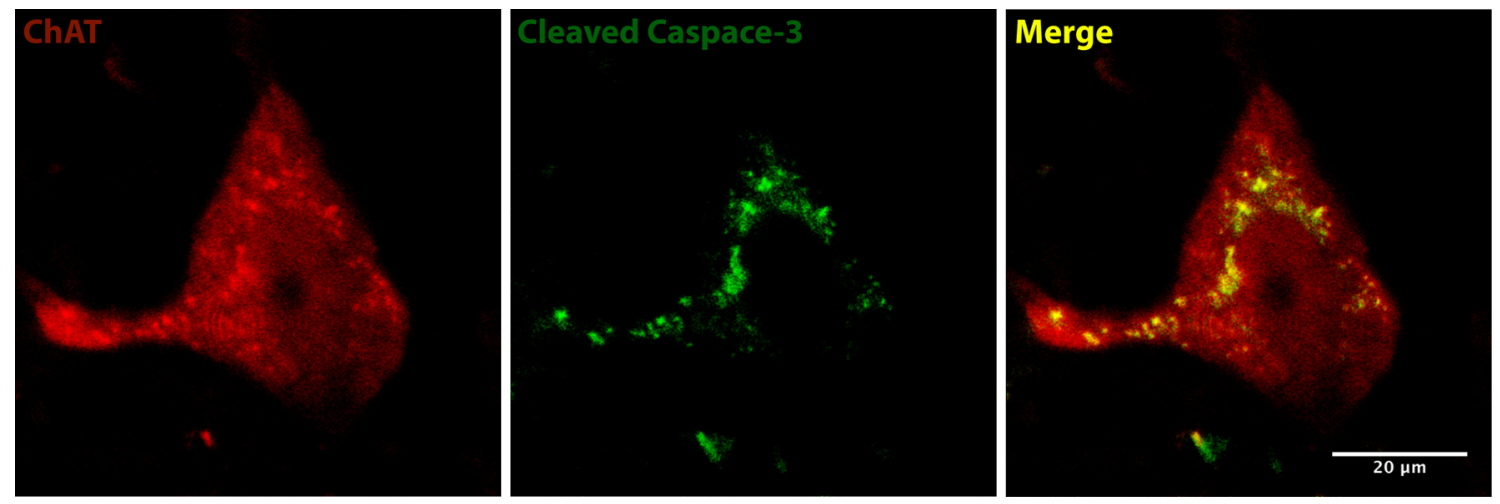

Fig. 2 Chronic posterior cervical spinal cord induces apoptosis in motoneurons resides in the anterior horns of the gray matter. a Double immunofluorescence labeling with ChAT (red; motoneurons) and

neurological dysfunction appeared when compared to $\mathrm{Wt}$ CSM mice [29].

\section{Apoptotic cell death}

Apoptotic neurons and oligodendrocytes have been defined in human CSM suggesting that active cellular death mediates damage; a finding which adds to the development of the unique clinical picture of CSM [43]. Moreover, different studies have showed that neuronal and oligodendroglial cells undergo apoptosis even during the late stages of the induction of compression [27, 44]. Recently, Karadimas et al., using unbiased stereology techniques, measured the number of dead cells as well as the number of neuronal and oligodendrocyte cells undergoing active apoptosis in a 5-mm area centered on the compression epicenter. It was demonstrated that even up to 10 weeks following material implantation, numerous neurons and oligodendrocytes were undergoing apoptosis (Fig. 2). The ongoing oligodendrocytic apoptosis away from the compression epicenter was very well associated with axonal degeneration in the main corticospinal tracts indicating that apoptosis is implicated in the chronic demyelination of tracts away from the site of injury.

Although the tumor necrosis factor- $\alpha$, the mitogenactivated protein kinase pathways and the nuclear factor- $\kappa$ B [45] have been identified as potential apoptotic signaling pathways in CSM, there is also evidence that blocking the FasL signaling pathway results in decreased levels of cellular apoptosis and improved functional outcomes in CSM. It is obvious that only when identification of the extra and intracellular apoptotic pathways implicated in CSM is complete, we will be able to develop novel neuroprotective treatments for this disease. cleaved caspase-3 (green; apoptosis) demonstrate co-localization of two markers (Merge, yellow), indicating ongoing motoneuronal apoptosis in a CSM spinal cord tissue
Future research

Although many important steps have been take in recent years regarding gaining insight into CSM, there is still much to be researched in order that we might more fully understand the mechanisms involved. Uncovering the molecular pathways and anatomical reorganization in important neural networks under chronic compression of the cervical spinal cord will also help to direct endeavors to develop new therapeutic strategies. Extensive behavioral research linked with neuroanatomical findings in models of CSM will inform the "bedside"; a fact which will facilitate the development of sensitive clinical measures which will reflect the clinical presentation of CSM and allow for early detection in mild states of the disease.

In addition, further research on understanding the pathophysiology could lead to new therapeutic targets and the identification of new pharmaceutical therapies that might eliminate the potential complications of surgical decompression as well as augment its benefits. As an encouraging example, the current clinical CSMProtect clinical trial (www.clinicatrials.gov reference: NCT01257828 [3]) was designed using information from a basic science research study examining the combination of riluzole (pre-, peri- and post-operative administration) and surgical decompression in preclinical experimental models of CSM [46].

\section{Conclusion}

In conclusion, in recent years basic research science has made large strides in identifying the physiological phenomena triggered by the static and dynamic load on the cervical spinal cord in CSM. However, the complex cascade of biomolecular events as a result of the unique 
pathophysiology has yet to be fully elucidated. As the events involved become clearer, accessible targets for neuroprotective or other therapeutic targets may be identified to improve outcomes in CSM and after surgery for CSM.

\section{Conflict of interest None.}

Open Access This article is distributed under the terms of the Creative Commons Attribution License which permits any use, distribution, and reproduction in any medium, provided the original author(s) and the source are credited.

\section{References}

1. Kalsi-Ryan S, Karadimas SK, Fehlings MG (2013) Cervical spondylotic myelopathy: the clinical phenomenon and the current pathobiology of an increasingly prevalent and devastating disorder. Neuroscientist 19:409-421

2. Karadimas SK, Erwin WM, Ely CG et al (2013) Pathophysiology and natural history of cervical spondylotic myelopathy. Spine (Phila Pa 1976) 38:S21-S36

3. Fehlings MG, Wilson JR, Karadimas SK et al (2013) Clinical evaluation of a neuroprotective drug in patients with cervical spondylotic myelopathy undergoing surgical treatment: design and rationale for the CSM-protect trial. Spine (Phila Pa 1976) 38:S68-S75

4. Baptiste DC, Fehlings MG (2006) Pathophysiology of cervical myelopathy. Spine J 6:190S-197S

5. Fehlings MG, Skaf G (1998) A review of the pathophysiology of cervical spondylotic myelopathy with insights for potential novel mechanisms drawn from traumatic spinal cord injury. Spine (Phila Pa 1976) 23:2730-2737

6. Brain WR, Knight GC, Bull JW (1948) Discussion of rupture of the intervertebral disc in the cervical region. Proc R Soc Med 41:509-516

7. Mair WG, Druckman R (1953) The pathology of spinal cord lesions and their relation to the clinical features in protrusion of cervical intervertebral discs; a report of four cases. Brain 76:70-91

8. Taylor AR (1953) Mechanism and treatment of spinal-cord disorders associated with cervical spondylosis. Lancet 1:717-720

9. Crum B, Mokri B, Fulgham J (2000) Spinal manifestations of vertebral artery dissection. Neurology 55:304-306

10. Breig A, Turnbull I, Hassler O (1966) Effects of mechanical stresses on the spinal cord in cervical spondylosis. A study on fresh cadaver material. J Neurosurg 25:45-56

11. Gooding MR, Wilson CB, Hoff JT (1975) Experimental cervical myelopathy. Effects of ischemia and compression of the canine cervical spinal cord. J Neurosurg 43:9-17

12. Gooding MR, Wilson CB, Hoff JT (1976) Experimental cervical myelopathy: autoradiographic studies of spinal cord blood flow patterns. Surg Neurol 5:233-239

13. Doppman JL (1975) The mechanism of ischemia in anteroposterior compression of the spinal cord. Invest Radiol 10:543-551

14. McCormack BM, Weinstein PR (1996) Cervical spondylosis. An update. West J Med 165:43-51

15. Adams CB, Logue V (1971) Studies in cervical spondylotic myelopathy. II. The movement and contour of the spine in relation to the neural complications of cervical spondylosis. Brain 94:568-586

16. Strek P, Reron E, Maga P et al (1998) A possible correlation between vertebral artery insufficiency and degenerative changes in the cervical spine. Eur Arch Otorhinolaryngol 255:437-440
17. Uchida K, Nakajima H, Sato R et al (2009) Cervical spondylotic myelopathy associated with kyphosis or sagittal sigmoid alignment: outcome after anterior or posterior decompression. J Neurosurg Spine 11:521-528

18. Shimizu K, Nakamura M, Nishikawa Y et al (2005) Spinal kyphosis causes demyelination and neuronal loss in the spinal cord: a new model of kyphotic deformity using juvenile Japanese small game fowls. Spine (Phila Pa 1976) 30:2388-2392

19. Shamji MF, Ames CP, Smith JS et al (2013) Myelopathy and spinal deformity: relevance of spinal alignment in planning surgical intervention for degenerative cervical myelopathy. Spine (Phila Pa 1976) 38:S147-S148

20. Kurokawa R, Murata H, Ogino M et al (2011) Altered blood flow distribution in the rat spinal cord under chronic compression. Spine (Phila Pa 1976) 36:1006-1009

21. Good DC, Couch JR, Wacaser L (1984) "Numb, clumsy hands" and high cervical spondylosis. Surg Neurol 22:285-291

22. Al-Mefty O, Harkey HL, Marawi I et al (1993) Experimental chronic compressive cervical myelopathy. J Neurosurg 79:550-561

23. Hoff JNM, Pitts L, Vilnis V, Tuerk K, Lagger R (1977) The role of ischemia in the pathogenesis of cervical spondylotic myelopathy: a review and new microangiopathic evidence. Spine (Phila Pa 1976) 2:100-108

24. Ono KOH, Tada K, Yamamoto T (1977) Cervical myelopathy secondary to multiple spondylotic protrusions. Spine (Phila Pa 1976) $2: 109-125$

25. Ogino H, Tada K, Okada K et al (1983) Canal diameter, anteroposterior compression ratio, and spondylotic myelopathy of the cervical spine. Spine (Phila Pa 1976) 8:1-15

26. Carlson GD, Warden KE, Barbeau JM et al (1997) Viscoelastic relaxation and regional blood flow response to spinal cord compression and decompression. Spine (Phila Pa 1976) 22:1285-1291

27. Karadimas SK, Moon E, Yu W, Satkunendrarajah K, Kallitsis JK, Gatzounis G, Fehlings MG (2013) A novel experimental model of cervical spondylotic myelopathy (CSM) to facilitate translational research. neurobiology of disease. Neurobiol Dis 54:43-58

28. Klironomos G, Karadimas S, Mavrakis A et al (2011) New experimental rabbit animal model for cervical spondylotic myelopathy. Spinal Cord 49:1097-1102

29. Yu W, Karadimas S, Fehlings MG (2012) Human and animal model evidence supporting a role for $\mathrm{Cx} 3 \mathrm{cr} 1$. In: Mediating the inflammatory response in cervical spondylotic myelopathy. Abstract presented at the 2012 Society for Neuroscience Meeting in October, New Orleans, Society of neuroscience

30. Noble LJ, Wrathall JR (1989) Distribution and time course of protein extravasation in the rat spinal cord after contusive injury. Brain Res 482:57-66

31. Loy DN, Crawford CH, Darnall JB et al (2002) Temporal progression of angiogenesis and basal lamina deposition after contusive spinal cord injury in the adult rat. J Comp Neurol 445:308-324

32. Fleming JC, Norenberg MD, Ramsay DA et al (2006) The cellular inflammatory response in human spinal cords after injury. Brain 129:3249-3269

33. Noble LJ, Donovan F, Igarashi T et al (2002) Matrix metalloproteinases limit functional recovery after spinal cord injury by modulation of early vascular events. J Neurosci 22:7526-7535

34. Schnell L, Fearn S, Schwab ME et al (1999) Cytokine-induced acute inflammation in the brain and spinal cord. J Neuropathol Exp Neurol 58:245-254

35. Strbian D, Durukan A, Pitkonen M et al (2008) The blood-brain barrier is continuously open for several weeks following transient focal cerebral ischemia. Neuroscience 153:175-181

36. Nico B, Ribatti D (2012) Morphofunctional aspects of the bloodbrain barrier. Curr Drug Metab 13:50-60 
37. Yu WR, Liu T, Kiehl TR, Fehlings MG (2011) Human neuropathological and animal model evidence supporting a role for Fas-mediated apoptosis and inflammation in cervical spondylotic myelopathy. Brain 134:1277-1292

38. Harrison JK, Jiang Y, Chen S et al (1998) Role for neuronally derived fractalkine in mediating interactions between neurons and CX3CR1-expressing microglia. Proc Natl Acad Sci USA 95:10896-10901

39. Tarozzo G, Campanella M, Ghiani M et al (2002) Expression of fractalkine and its receptor, CX3CR1, in response to ischaemiareperfusion brain injury in the rat. Eur J Neurosci 15:1663-1668

40. Denes A, Ferenczi S, Halasz J et al (2008) Role of CX3CR1 (fractalkine receptor) in brain damage and inflammation induced by focal cerebral ischemia in mouse. J Cereb Blood Flow Metab 28:1707-1721

41. Fuhrmann M, Bittner T, Jung CK et al (2010) Microglial Cx3cr1 knockout prevents neuron loss in a mouse model of Alzheimer's disease. Nat Neurosci 13:411-413

42. Fumagalli S, Perego C, Ortolano F, De Simoni MG (2013) CX3CR1 deficiency induces an early protective inflammatory environment in ischemic mice. Glia 61:827-842
43. Karadimas SK, Gialeli CH, Klironomos G et al (2010) The role of oligodendrocytes in the molecular pathobiology and potential molecular treatment of cervical spondylotic myelopathy. Curr Med Chem 17:1048-1058

44. Yu WR, Baptiste DC, Liu T et al (2009) Molecular mechanisms of spinal cord dysfunction and cell death in the spinal hyperostotic mouse: implications for the pathophysiology of human cervical spondylotic myelopathy. Neurobiol Dis 33:149-163

45. Karadimas SK, Klironomos G, Papachristou DJ et al (2013) Immunohistochemical profile of NF-kappaB/p50, NF-kappaB/ p65, MMP-9, MMP-2, and u-PA in experimental cervical spondylotic myelopathy. Spine (Phila Pa 1976) 38:4-10

46. Karadimas SKME, Fehlings MG (2012) The sodium channel/ glutamate blocker riluzole is complementary to decompression in a preclinical experimental model of cervical spondylotic myelopathy (CSM): implications for translational clinical application. Neurosurgery $71: 543$ 